\title{
Use of antagonists and morpholinos in loss-of-function analyses: estrogen receptor ESR2a mediates the effects of 17alpha-ethinylestradiol on primordial germ cell distribution in zebrafish
}

\author{
Jingying $\mathrm{Hu}$, Shaoyang Sun, Meng Guo and Houyan Song*
}

\begin{abstract}
Background: Various chemicals released into the aquatic environment adversely affect the reproductive system of fish, particularly by changing gonad structure and function. 17alpha-ethinylestradiol (EE2) is a potent environmental estrogen that disrupts sexual differentiation and normal reproduction in fish. Previous studies have shown that exposure to endocrine-disrupting chemicals (EDCs) disrupts the migration of primordial germ cells (PGCs) in zebrafish.

Methods: To investigate the effects of EE2 exposure on PGC migration, zebrafish embryos were injected with gfp-nanos mRNA to label PGCs and subsequently exposed to different concentrations of EE2. Typical estrogen receptor antagonist treatment and morpholino knockdown experiments were used to identify functional estrogen receptors that mediate the effects of EE2.

Results: The migration of PGCS was disrupted after exposure to high concentrations of EE2 (1 mirog/L). Loss-of-function analyses were performed for estrogen receptor ESR1, ESR2a, and ESR2b, and only loss of ESR2a resulted in a decreased number of ectopic PGCs following exposure to 1 mirog/L EE2.

Conclusions: EE2 exposure disrupts PGC migration and distribution, and this effect is mediated through the estrogen receptor ESR2a.
\end{abstract}

Keywords: 17alpha-ethinylestradiol, Primordial germ cells, Estrogen receptor

\section{Background}

The gonads are the primary targets of environmental pollutant toxicity. The mechanisms underlying defective gonadal development after exposure to pollutants have not been fully elucidated, and primordial germ cells (PGCs) may be the primary targets [1]. PGCs are progenitors of germ cells that migrate to the location of gonad development within 24 hours post fertilization (hpf). Recent studies have shown that nanos gene expression is a definitive marker of primordial germ cells in early zebrafish embryos $[2,3]$. To visualize PGCs, the coding sequence of green fluorescent protein (GFP) was fused to the 3 ' un-translated region (3'UTR) of zebrafish nanos1 mRNA, and mRNA

\footnotetext{
* Correspondence: hysong@shmu.edu.cn

Department of Biochemistry and Molecular Biology, Shanghai Medical School and Key Laboratory of Molecular Medicine, Ministry of Education, Fudan University, Shanghai 200032, PR China
}

transcribed from this construct was subsequently injected into zebrafish embryos [2].

17alpha-ethinylestradiol (EE2) is a potent environmental estrogen that has been shown to disrupt sexual differentiation and reproduction. The effects of EE2 are mediated through the transcriptional activities of the nuclear estrogen receptors, ESR1 and ESR2. Upon binding to a ligand in the nucleus, ESR1 and ESR2 bind to a specific estrogen response element (ERE) in the promoters of target genes. Zebrafish have a single esr 1 gene and two esr2 genes, which encode ESR1, ESR2a, and ESR2b, respectively. Menuet et al. [4] showed differential regulation of ESR1, ESR2a, and ESR2b after exposure to estradiol-17beta. ICI, an estrogen receptor antagonist (ER-antagonist), blocks estrogen activity through two ER subtypes, ESR1 and ESR2 [5], and shows little selectivity in its activation of these receptors. Sun et al. [6] 
identified the estrogen receptor antagonist methylpiperidino-pyrazole (MPP), which is ESR1-selective. Subsequently, Compton et al. [7] identified the potent and efficient ESR2 antagonist pyrazolo [1,5-a]pyrimidine to 2- phenyl -3- (4-hydroxyphenyl) -5,7- bis (trifluoromethyl) -pyrazolo [1,5-a] pyrimidine (PHTPP), which has minimal effects on ESR1.

In the present study, we examined the effects of EE2 exposure on the distribution of primordial germ cells in zebrafish embryos and characterized the roles of each estrogen receptor during this process. Exposure to $1 \mu \mathrm{g} / \mathrm{L}$ EE2 adversely affected the primordial germ cell distribution prior to gonad formation, and ESR2a played an important role in this process. These results may provide insight into the gonadal abnormalities observed in previous studies.

\section{Methods}

\section{Zebrafish strain and maintenance}

Wild-type zebrafish ( $\mathrm{AB}^{*}$ strain) were obtained from the Zebrafish International Resource Center (ZIRC, Oregon, USA). Embryos were collected following natural spawning. Wild-type zebrafish were raised, maintained, and staged as previously described [8]. In some cases, embryos and larvae were initially raised in water containing $0.2 \mathrm{mM} 1$ phenyl-2-thio-urea (PTU) to prevent pigment formation.

\section{Plasmid constructs}

The gfp-nanos-3'UTR construct contained the GFP ORF fused to the 3'UTR of nanos. The 3'UTR of nanos was cloned from zebrafish cDNA using specific primers (Table 1). The GFP ORF was cloned from the vector pEGFP-1 (BD Biosciences Clontech, USA) using specific primers (Table 1). The amplified fragments were cloned into the pGEM-T vector (Promega, USA) using the restriction enzyme SacII.

pEGFP-N1-esr $2 a$ is a construct fusing the 5'UTR region of the esr $2 a$ gene to GFP to act as a reporter for morpholino knockdown effectiveness, so is pEGFP-N1esr $2 b$. $p E G F P-N 1$-esr $2 a$ and $p E G F P-N 1$-esr $2 b$ were constructed using specific primers listed in Table 1 . The primers were designed based on the $5^{\prime}$-terminal sequence surrounding the putative start codons of zebrafish esr $2 a$ [Ensembl Transcript ID: ENSDART00000131069] and $e s r 2 b$ [Ensembl Transcript ID: ENSDART00000131800]. In total, $299 \mathrm{bp}$ of the 5'UTR region of the esr $2 a$ gene and $256 \mathrm{bp}$ of the $5^{\prime} \mathrm{UTR}$ region of the $e s r 2 b$ gene were PCR-amplified and cloned into the pEGFP-N1 vector (BD Biosciences Clontech, USA) at the BglI and BamHI restriction enzyme sites.

\section{Microinjection}

For injection, mRNA was prepared using the mMessage mMachine kit (Ambion, USA). RNA was diluted in $10 \mathrm{mM}$ HEPES (pH 7.6) and microinjected into zebrafish embryos at the one-to-four cell stage (200-400 pg/embryo).

Morpholino (MO) antisense oligonucleotides targeting the $5^{\prime}$ UTR region of each gene were obtained from Gene Tools, LLC (USA). The following MO sequences were used: esr2a-MO: $5^{\prime}$-TGTCTCCTTCGGGATAC TCGGACAT-3' and esr $2 b$-MO: 5'-AGCTCATGCT GGAGAACACAAGAGA-3'. One- and two-cell-stage wild-type zebrafish embryos were injected with MOs (2-5 ng/embryo).

The $p E G F P$-N1-esr2a and $p E G F P$-N1-esr $2 b$ plasmid constructs were prepared using a miniprep kit (Qiagen, USA). The plasmids were injected into one-cell-stage embryos at $5 \mathrm{ng} /$ embryo (stock concentration of $50 \mu \mathrm{g} / \mathrm{L}$ DNA in $100 \mathrm{mM} \mathrm{KCl}$ containing $0.02 \%$ phenol red).

\section{Microscopy and imaging}

The embryos and larvae were examined using an Olympus SZX12 microscope (Olympus, Japan), with or without a GFP filter, and photographed using a DP70 digital camera (Olympus, Japan).

\section{EE2 exposure and ER-antagonist co-exposure}

Stock solutions of EE2 were prepared in $100 \%$ ethanol, and the exposure concentration were $1 \mathrm{ng} / \mathrm{L}, 10 \mathrm{ng} / \mathrm{L}$, $100 \mathrm{ng} / \mathrm{L}, 500 \mathrm{ng} / \mathrm{L}, 1 \mu \mathrm{g} / \mathrm{L}$ and $2 \mu \mathrm{g} / \mathrm{L}$. Stock solutions of ICI (ER antagonist), MPP (ESR1 antagonist), and PHTPP (ESR2 antagonist; Tocris, UK) were prepared in $100 \%$ ethanol. Each ER-antagonist was used at a concentration of $1 \mu \mathrm{g} / \mathrm{L}$. Control fish were exposed to $1 \mu \mathrm{l} / \mathrm{L}$ ethanol. Every exposure group contained at least 100 chorionic embryos, and each experiment included four replicates for each treatment. After exposure for $24 \mathrm{~h}$, the PGC distribution in each embryo was examined.

Table 1 All primers used in this article

\begin{tabular}{|c|c|c|}
\hline & \multicolumn{2}{|c|}{ Primers } \\
\hline & Sense & Antisense \\
\hline nanos-3'UTR & 5'-GGAATTCAAAGCGCACACCAAGAGATT-3' & 5'-TCCCCGCGGAATGTTTATATTTTCCTCACATTTTC-3' \\
\hline pEGFP-N1 & 5'-ATGGTGAGCAAGGGCGAGGA-3' & 5'-TTACTTGTACAGCTCGTCCA-3' \\
\hline esr2a & 5'-GAAGATCTCACTGAGGAGTATCGAGGAC-3' & 5'-TTGGATCCCAGAGCGGGACTGTAAAA-3' \\
\hline$e s r 2 b$ & 5'-GAAGATCTAGTTGGGCCTGAGATGCA-3' & 5'-TTGGATCCTTAGGGCTCCGTGGTTGA-3' \\
\hline
\end{tabular}




\section{Statistical analysis}

The statistical software SPSS 18.0 was used to calculate the normality and homogeneity of variance of the data for embryos with ectopic PGCs. One-way analysis of variance (ANOVA) followed by Tukey's test was used to identify differences in the percentage of embryos with ectopic PGCs among the various treatment conditions.

\section{Results}

\section{Effects of EE2 on PGC distribution along the anterior-} posterior axis

We observed GFP expression in developing embryos after microinjection with gfp-nanos-3'UTR mRNA. GFP was expressed from the blastula stage in all injected embryos. At the $50 \%$ to $80 \%$ epiboly stage, a few cells showed stronger fluorescence than the surrounding cells, and these cells were referred to as brighter cells. The migration routes and final location of the brighter cells were similar to those described as PGCs in the zebrafish embryo $[9,10]$. To assess the impact of EE2 on PGC distribution, the embryos were injected with the $g f p$ nanos-3'UTR mRNA and were immediately exposed to different concentrations of EE2 till $24 \mathrm{hpf}$, when the cells are clustered along either side of the embryonic midline. At $24 \mathrm{hpf}$, PGCs were located below the somites and dorsal to the anterior end of the yolk extension in the non-EE2-treated group (Figure 1A). After 24 hours of EE2 treatment, the morphology of all embryos was normal, although some embryos displayed an abnormal PGC distribution pattern, in which ectopic PGCs were observed at the midbrain-hindbrain boundary and along the branchial arch at the $24 \mathrm{hpf}$ stage, similar to the spadetail mutant [10] (Figure 1B). After exposure to 500 ng/L EE2, 13.5\% of embryos displayed ectopic PGCs. In the 1 and $2 \mu \mathrm{g} / \mathrm{L}$ EE2 exposure groups, approximately $20 \%$ of the embryos showed ectopic PGCs. Ectopic
PGCs were observed on the back or abdomen, or along the branchial arches of these embryos (Figure 1C). The percentage of ectopic PGCs in the EE2-treated groups was significantly higher than that in the other groups $(\mathrm{p}<0.01$, one-way ANOVA, followed by Tukey's test) (Figure 2).

\section{Effects of estrogen receptor inhibition or knockdown on EE2-induced ectopic PGC distribution}

To assess the involvement of estrogen receptors in the effect of EE2 on PGC distribution, typical estrogen receptor antagonists and specific estrogen receptor morpholinos were used. ICI was used to inhibit all ERs, MPP was used to inhibit ESR1, PHTPP was used to inhibit ESR2, and the esr $2 a$ and esr $2 b$ morpholinos were used to knock down the expression of ESR2a and ESR2b, respectively.

To confirm the efficacy of the morpholino approach, esr $2 a-\mathrm{MO}$ and esr $2 b-\mathrm{MO}$ were co-injected with a green fluorescent protein (GFP) reporter containing the partial 5'UTR and start codons of esr $2 a$ and esr $2 b$, separately. At $7 \mathrm{hpf}$, the esr $2 a-\mathrm{MO}$ specifically knocked down the expression of GFP from the associated RNA transcript in $100 \%$ of embryos $(n=34)$ (Figure 3C). In contrast, GFP was highly expressed in the group of control embryos injected with only the plasmid $(n=29)$ (Figure 3A). These results confirmed the ability of the esr $2 a-\mathrm{MO}$ to block the translation of the target protein sequence. Similar results were observed with the esr $2 b-\mathrm{MO}$ (Figure 3B and D).

In most of the control non-EE2 treated embryos, PGCs arrived at their destination where the future gonad develops by $24 \mathrm{hpf}$ (Figure 1 ). In contrast, ICI or MPP treatment disrupted PGC distribution in $14 \%$ and $10 \%$ of zebrafish embryos, respectively (Figure 4). Surprisingly, treatment with PHTPP, esr $2 a-\mathrm{MO}$ or esr $2 b-\mathrm{MO}$ alone did not disrupt PGC distribution (Figure 4). These
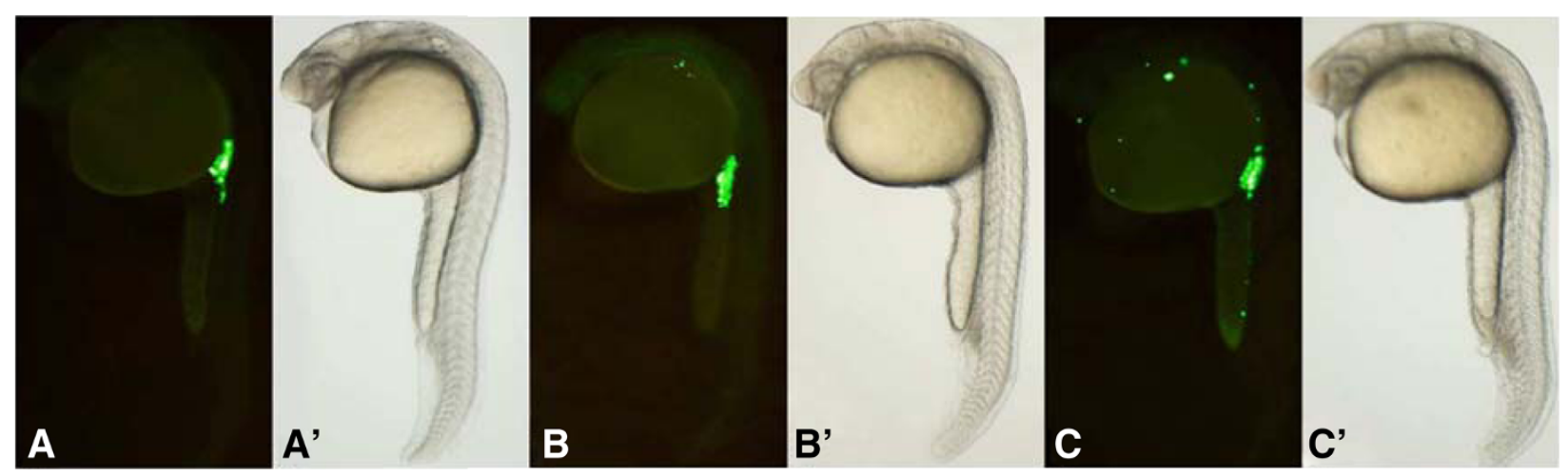

Figure 1 Fluorescence images of zebrafish PGCs after exposure to various concentrations of EE2. A: In all treatment groups, normal PGCS were observed in the anterior region of the yolk extension at $24 \mathrm{hpf}$. B: In the $1 \mathrm{ng} / \mathrm{L}, 10 \mathrm{ng} / \mathrm{L}$, and $100 \mathrm{ng} / \mathrm{L}$ EE2 exposure groups, ectopic PGCs were primarily observed along the branchial arch, similarly to the spadetail mutant. C: In the $500 \mathrm{ng} / \mathrm{L}, 1 \mu \mathrm{g} / \mathrm{L}$ and $2 \mu \mathrm{g} / \mathrm{L}$ EE2 exposure groups, many ectopic PGCs were observed along the branchial arch, on the back, in the abdomen, and along the trunk. $\mathbf{A}^{\prime}, \mathbf{B}^{\prime}$, and $\mathbf{C}^{\prime}$ : Bright-field views show the morphology of the embryos. 


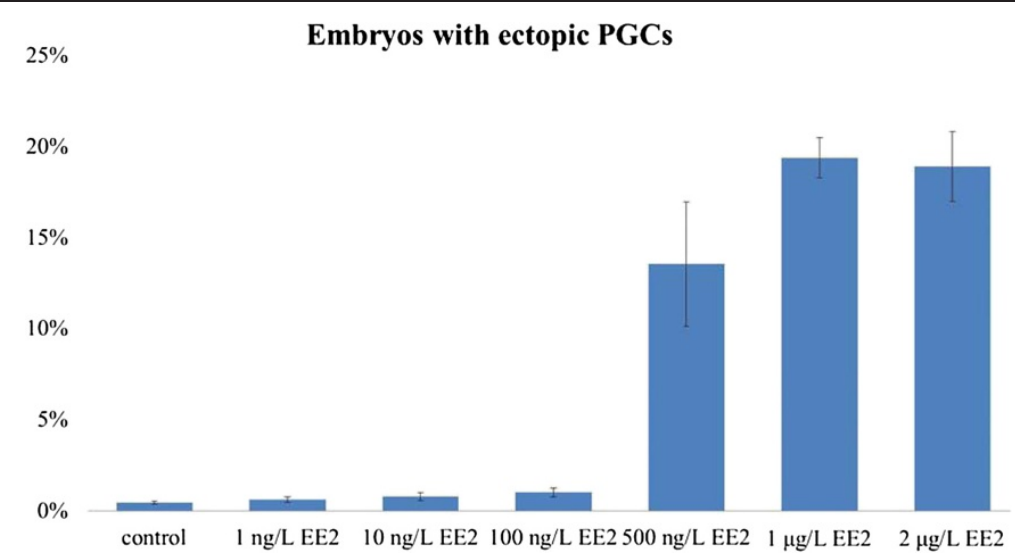

Figure 2 Percentage of zebrafish embryos with ectopic PGCs in different exposure groups. In the control, $1 \mathrm{ng} / \mathrm{L}, 10 \mathrm{ng} / \mathrm{L}$, and 100 ng/L EE2 exposure groups, less than $2 \%$ of the zebrafish embryos showed ectopic PGCs. In the $500 \mathrm{ng} / \mathrm{L}$ EE2 exposure group, approximately $13 \%$ of zebrafish embryos contained ectopic PGCs. In the $1 \mu \mathrm{g} / \mathrm{L}$ EE2 exposure group, approximately 20\% of zebrafish embryos contained ectopic PGCs. In the $2 \mu \mathrm{g} / \mathrm{L}$ EE2 exposure group, approximately $19 \%$ of zebrafish embryos contained ectopic PGCs. These rates were significantly higher than that of other groups ( $p<0.01$, one-way ANOVA followed by Tukey's test).

results suggest that instead of ESR2, only ESR1 is required for normal PGC development.

In all of the $1 \mu \mathrm{g} / \mathrm{L}$ EE2 treated embryos, the gross morphology are normal. However, $1 \mu \mathrm{g} / \mathrm{L}$ EE2 treatment alone resulted in ectopic PGC in 19\% of embryos. Compared to the $1 \mu \mathrm{g} / \mathrm{L}$ EE2 group, approximately $37 \%$ and $40 \%$ embryos showed ectopic PGCs after co-exposure to $1 \mu \mathrm{g} / \mathrm{L} \mathrm{EE} 2$ and $1 \mu \mathrm{g} / \mathrm{L}$ ICI or $1 \mu \mathrm{g} / \mathrm{L}$ EE2 and $1 \mu \mathrm{g} / \mathrm{L}$ MPP, respectively. Addition of PHTPP or esr2a-MO in $1 \mu \mathrm{g} / \mathrm{L}$ EE2-treated groups resulted in fewer embryos with ectopic PGCs (approximately 13\% and 11\%, respectively), suggesting the adverse effect of EE2 on PGC distribution is mediated through ESR2a. Interestingly, injection of esr $2 b$-MO in $1 \mu \mathrm{g} / \mathrm{L}$ EE2-treated embryos has no effect on PGC distribution compared to EE2 treatment alone, as shown by the number of embryos with ectopic PGCs are similar between the two groups. This result indicates that ESR2b does not mediate the effect of EE2 on PGC distribution (Figure 4).

\section{Discussion}

In 2001, Koprunner et al. [2] cloned and identified the nanos gene as a marker of PGCs. The nanos mRNA is maternally loaded and plays an important role in PGC survival and migration. In the 3'UTR of nanos mRNA, a conserved sequence protects the mRNA from degradation in the PGCs [11]. In the present study, we fused the GFP coding region to the nanos-3'UTR. The translated GFP
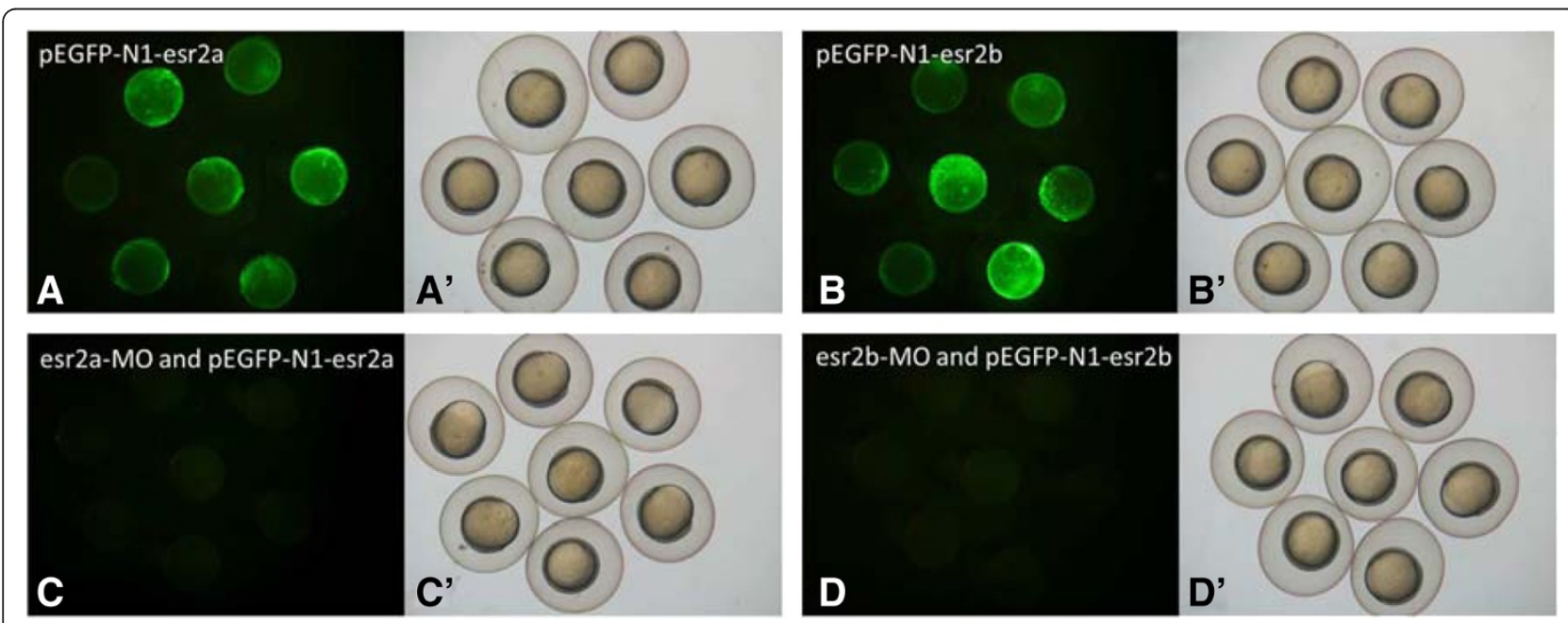

Figure 3 Effects of MO on GFP expression in 7 hpf embryos injected with recombinant plasmids. A, B: A mosaic pattern of GFP expression was detected throughout the embryos. C, D: GFP expression was undetectable after co-injection with MO and recombinant plasmids. $\mathbf{A}^{\prime}, \mathbf{B}^{\prime}, \mathbf{C}^{\prime}, \mathbf{D}^{\prime}$ : Bright-field views show the morphology of the embryos. 


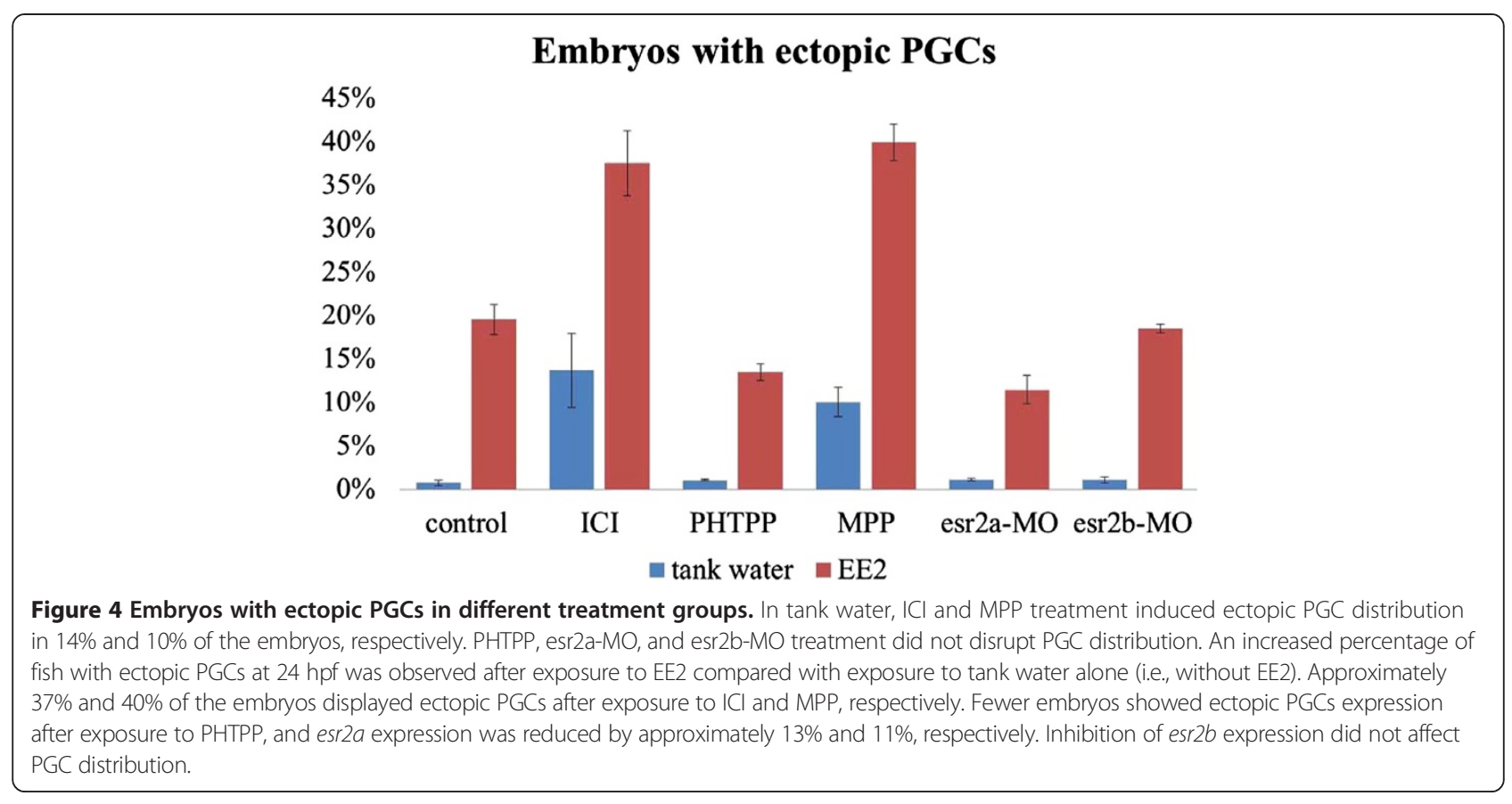

protein labeled PGCs with green fluorescence, as previously observed in somatic cells $[3,12]$.

Previous studies have shown that environmental endocrine disruptors and estrogens affect the development and migration of fish PGCs and the development of gonads, thereby affecting reproductive function $[1,13,14]$. In the present study, we demonstrated that EE2 treatment disrupts PGC distribution in zebrafish and that ESR2a might play a role in this effect. EE2 exposure interferes with PGC distribution at a concentration range of $100-500 \mathrm{ng} / \mathrm{L}$. Notably, higher doses of EE2 (>1 $\mu \mathrm{g} / \mathrm{L})$ did not produce stronger phenotypes.

PGC migration in zebrafish is well understood, particularly because guidance by the chemokine SDF-1a [15] and its receptor CXCR4b $[15,16]$ has been characterized. CXCR7 is another receptor for SDF-1 $[17,18]$ that promotes cell migration [17]. CXCR7 is crucial for proper migration of PGCs towards targets in the somatic environment rather than within migrating cells [19]. SDF1/CXCR4/CXCR7 signaling also participates in the migration of the posterior lateral line primordium, generating the embryonic posterior lateral line in zebrafish $[20,21]$. The estrogen receptor ESR1 represses the chemokine receptor CXCR4 and thus controls cell migration in the zebrafish posterior lateral line system [22]. Both the inactivation and overexpression of ESR1 result in aborted migration, confirming the importance of this receptor in SDF1-guided migration [22]. The synergistic effects of ICI or MPP and EE2 may explain the increased percentage of zebrafish with ectopic PGCs after ESR1 and all ERs were inhibited. Importantly, knocking down esr $2 a$ reduced the percentage of embryos with ectopic
PGCs after exposure to high concentration of EE2, suggesting ESR2a may mediate the effects of EE2 on PGC migration. PHTPP inhibited ESR2, including both ESR2a and ESR2b, and this effect may have contributed to the weaker phenotype observed after co-exposure of PHTPP and EE2.

Functional gonad formation depends on the migration of PGCs from specification sites to locations of future gonad development [23-25]. This migration requires a chemokine micro-environment in which SDF-1a is secreted from somatic cells. The effects of EE2 on zebrafish PGC migration may reflect either changes in the PGC response to chemokines or interference of the micro-environment in which PGC migration occurs. Thus, the effects of EE2 on zebrafish PGC migration may be mediated by ESR2a, but the specific expression of ESR2a in PGCs or somatic cells as EE2 target sites remains to be determined.

\section{Conclusions}

The present study showed that the migration and distribution of PGCs is affected after exposure to high doses of EE2 (500 ng/L), and approximately $20 \%$ of embryos displayed ectopic PGCs. Knocking down esr $2 a$ or application of a ESR2 inhibitor PHTPP, reduced the percentage of embryos with ectopic PGCs significantly after exposure to high doses of EE2, indicating that ESR2a may play an important role in EE2-related PGC phenotypes.

\section{Abbreviations}

EDCs: Endocrine disrupting chemicals; EE2: 17a-ethinylestradiol;

PGCs: Primordial germ cells; hpf: Hours post fertilization; GFP: Green fluorescent protein; 3' UTR: 3' un-translated region; ERE: Estrogen response element; ER: Estrogen receptor; MPP: Methyl-piperidino-pyrazole; 
PHTPP: Pyrazolo [1,5-a]pyrimidine to 2- phenyl -3- (4-hydroxyphenyl) -5,7bis (trifluoromethyl) -pyrazolo [1,5-a] pyrimidine; PTU: 1-phenyl-2-thio-urea; MO: Morpholino; ANOVA: A one-way analysis of variance.

\section{Competing interests}

The authors declare that they have no competing interests.

\section{Authors' contributions}

JYH participated in the design of the study, and drafted the manuscript. JYH and SYS conducted the data collection. JYH and MG participated in the statistical analysis, and performed the diagramming. HYS conceived of the study, participated in its design and coordination, and helped to draft the manuscript. All the authors read and approved the final manuscript.

\section{Acknowledgements}

The authors would like to thank Dr. Hui Xu of molecular and behavioral neuroscience institute, University of Michigan, USA, and Dr. Yu-Sheng Liu of Centre for Reproductive Medicine, Department of Obstetrics and Gynecology, Anhui Provincial Hospital affiliated with Anhui Medical University, Hefei, Anhui 230000, China, for revising the manuscript. The English usage has been revised by the English language professional service: http://www.aje.com/.

Received: 19 November 2013 Accepted: 10 May 2014

Published: 15 May 2014

\section{References}

1. Willey JB, Krone PH: Effects of endosulfan and nonylphenol on the primordial germ cell population in pre-larval zebrafish embryos. Aquat Toxicol 2001, 54:113-123.

2. Koprunner M, Thisse C, Thisse B, Raz E: A zebrafish nanos-related gene is essential for the development of primordial germ cells. Genes Dev 2001, 15:2877-2885.

3. Saito T, Fujimoto T, Maegawa S, Inoue K, Tanaka M, Arai K, Yamaha E: Visualization of primordial germ cells in vivo using GFP-nos1 $3^{\prime} \mathrm{UTR}$ mRNA. Int J Dev Biol 2006, 50:691-699.

4. Menuet A, Le Page Y, Torres O, Kern L, Kah O, Pakdel F: Analysis of the estrogen regulation of the zebrafish estrogen receptor (ER) reveals distinct effects of ERalpha, ERbeta1 and ERbeta2. J Mol Endocrinol 2004, 32:975-986.

5. Wakeling AE, Bowler J: ICI 182,780, a new antioestrogen with clinical potential. J Steroid Biochem Mol Biol 1992, 43:173-177.

6. Sun J, Huang YR, Harrington WR, Sheng S, Katzenellenbogen JA, Katzenellenbogen BS: Antagonists selective for estrogen receptor alpha. Endocrinology 2002, 143:941-947.

7. Compton DR, Sheng S, Carlson KE, Rebacz NA, Lee IY, Katzenellenbogen BS, Katzenellenbogen JA: Pyrazolo[1,5-a]pyrimidines: estrogen receptor ligands possessing estrogen receptor beta antagonist activity. J Med Chem 2004, 47:5872-5893.

8. Hu J, Sun S, Jiang Q, Wang W, Gui Y, Song H: Yes-associated protein (yap) is required for early embryonic development in zebrafish (danio rerio). Int J Biol Sci 2013, 9:267-278.

9. Yoon C, Kawakami K, Hopkins N: Zebrafish vasa homologue RNA is localized to the cleavage planes of 2- and 4-cell-stage embryos and is expressed in the primordial germ cells. Development 1997, 124:3157-3165.

10. Weidinger $\mathrm{G}$, Wolke $\mathrm{U}$, Koprunner M, Klinger M, Raz E: Identification of tissues and patterning events required for distinct steps in early migration of zebrafish primordial germ cells. Development 1999, 126:5295-5307.

11. Giraldez AJ, Mishima Y, Rihel J, Grocock RJ, Van Dongen S, Inoue K, Enright AJ, Schier AF: Zebrafish MiR-430 promotes deadenylation and clearance of maternal mRNAs. Science 2006, 312:75-79.

12. Yoshizaki G, Tago Y, Takeuchi Y, Sawatari E, Kobayashi T, Takeuchi T: Green fluorescent protein labeling of primordial germ cells using a nontransgenic method and its application for germ cell transplantation in salmonidae. Biol Reprod 2005, 73:88-93.

13. Weber LP, Hill RL Jr, Janz DM: Developmental estrogenic exposure in zebrafish (Danio rerio): II. Histological evaluation of gametogenesis and organ toxicity. Aquat Toxicol 2003, 63:431-446.

14. Hill RL Jr, Janz DM: Developmental estrogenic exposure in zebrafish (Danio rerio): I. Effects on sex ratio and breeding success. Aquat Toxicol 2003, 63:417-429.
15. Doitsidou M, Reichman-Fried M, Stebler J, Koprunner M, Dorries J, Meyer D, Esguerra CV, Leung T, Raz E: Guidance of primordial germ cell migration by the chemokine SDF-1. Cell 2002, 111:647-659.

16. Knaut H, Werz C, Geisler R, Nusslein-Volhard C: A zebrafish homologue of the chemokine receptor $\mathrm{Cxcr} 4$ is a germ-cell guidance receptor. Nature 2003, 421:279-282

17. Balabanian K, Lagane B, Infantino S, Chow KY, Harriague J, Moepps B, Arenzana-Seisdedos F, Thelen M, Bachelerie F: The chemokine SDF-1/ CXCL12 binds to and signals through the orphan receptor RDC1 in T lymphocytes. J Biol Chem 2005, 280:35760-35766.

18. Burns JM, Summers BC, Wang Y, Melikian A, Berahovich R, Miao Z, Penfold ME, Sunshine MJ, Littman DR, Kuo CJ, et al: A novel chemokine receptor for SDF-1 and I-TAC involved in cell survival, cell adhesion, and tumor development. J Exp Med 2006, 203:2201-2213.

19. Boldajipour B, Mahabaleshwar H, Kardash E, Reichman-Fried M, Blaser H, Minina S, Wilson D, Xu Q, Raz E: Control of chemokine-guided cell migration by ligand sequestration. Cell 2008, 132:463-473.

20. Dambly-Chaudiere C, Cubedo N, Ghysen A: Control of cell migration in the development of the posterior lateral line: antagonistic interactions between the chemokine receptors CXCR4 and CXCR7/RDC1. BMC Dev Biol 2007, 7:23.

21. Valentin G, Haas P, Gilmour D: The chemokine SDF1a coordinates tissue migration through the spatially restricted activation of $\mathrm{Cxcr} 7$ and Cxcr4b. Curr Biol 2007, 17:1026-1031.

22. Gamba L, Cubedo N, Ghysen A, Lutfalla G, Dambly-Chaudiere C: Estrogen receptor ESR1 controls cell migration by repressing chemokine receptor CXCR4 in the zebrafish posterior lateral line system. Proc Natl Acad Sci U S A 2010, 107:6358-6363.

23. Starz-Gaiano M, Lehmann R: Moving towards the next generation. Mech Dev 2001, 105:5-18.

24. Wylie C: Germ cells. Curr Opin Genet Dev 2000, 10:410-413.

25. Wylie C: Germ cells. Cell 1999, 96:165-174.

\section{doi:10.1186/1477-7827-12-40}

Cite this article as: Hu et al.: Use of antagonists and morpholinos in loss-of-function analyses: estrogen receptor ESR2a mediates the effects of 17alpha-ethinylestradiol on primordial germ cell distribution in zebrafish. Reproductive Biology and Endocrinology 2014 12:40.

\section{Submit your next manuscript to BioMed Central and take full advantage of:}

- Convenient online submission

- Thorough peer review

- No space constraints or color figure charges

- Immediate publication on acceptance

- Inclusion in PubMed, CAS, Scopus and Google Scholar

- Research which is freely available for redistribution 